\title{
ON SELF-INTERSECTION INVARIANTS
}

\author{
MARK GRANT \\ School of Mathematical Sciences, The University of Nottingham, University Park, \\ Nottingham NG7 2RD, United Kingdom \\ e-mail: Mark.Grant@nottingham.ac.uk
}

(Received 12 December 2011; accepted 27 March 2012; first published online 2 August 2012)

\begin{abstract}
We prove that the Hatcher-Quinn and Wall invariants of a selftransverse immersion $f: N^{n} \rightarrow M^{2 n}$ coincide. That is, we construct an isomorphism between their target groups, which carries one onto the other. We also employ methods of normal bordism theory to investigate the Hatcher-Quinn invariant of an immersion $f: N^{n} \rightarrow M^{2 n-1}$.

2010 Mathematics Subject Classification. Primary 57R42, 57R40, Secondary 57R19, 57R67.
\end{abstract}

1. Introduction. The problem of finding necessary and sufficient conditions for a given (smooth) immersion to be regularly homotopic to an embedding has been considered by many authors, going back to Whitney [17]. In favourable cases, complete obstructions can be given in terms of the self-intersection data of the immersion. This is true of Whitney's original trick, which shows that an immersion $f: N^{n} \rightarrow M^{2 n}$ with $M$ simply-connected is regularly homotopic to an embedding if and only if the algebraic sum of its double points is zero.

The non-simply-connected version of Whitney's trick was used by Wall [15] in the course of his pioneering work on surgery theory. To each immersion $f: N^{n} \rightarrow M^{2 n}$ (where $N$ is now assumed to be simply-connected, but $M$ not necessarily so), Wall describes a complete obstruction to the removal of double points. This invariant (which we denote by $\mu_{W}(f)$ below) lives in a certain quotient of the integral group ring of $\pi_{1}(M)$.

Meanwhile, Shapiro [13] and Haefliger [3] had set about generalising Whitney's trick to higher dimensional self-intersections, using deleted product constructions. Although their approach essentially reduces the problem to homotopy theory in the so-called meta-stable range, the invariants produced are rather difficult to compute. Later, Hatcher and Quinn [4] revisited the geometric constructions of Haefliger in the framework of bordism theory. They define, for each immersion $f: N^{n} \rightarrow M^{m}$, a regular homotopy invariant $\mu(f)$ in a certain normal bordism group. When $2 m \geq 3(n+1)$, the vanishing of $\mu(f)$ is a necessary and sufficient condition for $f$ to be regularly homotopic to an embedding.

The Hatcher-Quinn invariants have received relatively little attention in the literature (although see the papers of Klein and Williams [6, 7], Munson [9] and Salikhov [11]). This can perhaps be attributed to the difficulty of working directly with normal bordism groups, as well as the somewhat sketchy nature of the proofs in [4] 
(although more complete proofs have since been given by Klein and Williams using homotopy-theoretic methods, see [6, Appendix A]).

The current paper has two modest aims. Firstly, we identify the Hatcher-Quinn and Wall invariants of an immersion $f: N^{n} \rightarrow M^{2 n}$. Secondly, we offer some speculation as to what the analogue of Wall's invariant should be in the case of an immersion $f: N^{n} \rightarrow M^{2 n-1}$. We remark that in the case of an immersion $f: S^{n} \rightarrow \mathbb{R}^{2 n-1}$, a very satisfactory answer has been given by Ekholm [2] in terms of Smale invariants. Regular homotopy classes of immersions $f: M^{3} \rightarrow \mathbb{R}^{5}$ have been studied by Saeki-SzúcsTakase [10] and by Juhász [5]. In these dimensions, the presence of knotted spheres precludes the possibility of describing the regular homotopy class of an immersion in terms of its self-intersection data.

We now give the plan of the paper. After a brief review of normal bordism theory in Section 2, we review the definitions and results of Hatcher-Quinn and Wall in Sections 3 and 4, respectively. In Section 5, we prove the following precise result.

THEOREM A. Let $f: N^{n} \rightarrow M^{2 n}$ be a self-transverse immersion, where $N$ is closed and simply-connected and $M$ is connected. Then there is an isomorphism of abelian groups

$$
\mathcal{F}: H_{0}\left(\mathbb{Z}_{2} ; \mathbb{Z}[\pi]\right) \stackrel{\simeq}{\longrightarrow} \Omega_{0}\left(P(f, f)_{\mathbb{Z}_{2}} ; \zeta_{\mathbb{Z}_{2}}\right)
$$

under which $\mathcal{F}\left(\mu_{W}(f)\right)=\mu(f)$. That is, the Hatcher-Quinn and Wall invariants of $f$ coincide.

In Section 6, we use the Gysin sequence in normal bordism to study the HatcherQuinn invariant of an immersion $f: N^{n} \uparrow M^{2 n-1}$. We aim to construct an analogue of Wall's invariant, residing in a group defined in terms of the first and second homotopy groups of $M$, and depending only on the self-intersection data of $f$. The results in the final section go some way towards realising this goal.

My sincere thanks go to Andrew Ranicki, without whose considerable encouragement this paper would not have been written, and to the anonymous referee, for suggestions improving exposition.

2. Normal bordism. In this section, we collect some facts about normal bordism theory. These results may all be found in the paper of Salomonsen [12]. Alternative treatments have been given by Dax [1] and Koschorke [8]. For simplicity, we treat only the absolute bordism groups.

Let $X$ be a topological space, and let $\xi=\xi^{+}-\xi^{-}$be a virtual vector bundle over $X$ (we do not assume that $\xi^{+}$and $\xi^{-}$are of the same dimension). By an $n$ dimensional $\xi$-manifold over $X$, we mean a triple $\mathcal{M}=\left(M^{n}, f, F\right)$ consisting of a closed $n$-manifold $M^{n}$, a continuous map $f: M \rightarrow X$ and an equivalence class of vector bundle isomorphisms

$$
F: T M \oplus f^{*} \xi^{-} \oplus \varepsilon^{r} \stackrel{\simeq}{\longrightarrow} f^{*} \xi^{+} \oplus \varepsilon^{s},
$$

where $r$ and $s$ are the suitable integers (here and elsewhere $\varepsilon$ denotes a trivial bundle of the stated dimension). The equivalence relation is generated by stabilisation and homotopy of bundle isomorphisms. 
The negative of $\mathcal{M}$ is the triple $-\mathcal{M}=(M, f,-F)$ where

$$
-F=F \oplus(-1): T M \oplus f^{*} \xi^{-} \oplus \varepsilon^{r} \oplus \varepsilon^{1} \stackrel{\simeq}{\longrightarrow} f^{*} \xi^{+} \oplus \varepsilon^{s} \oplus \varepsilon^{1}
$$

If $\mathcal{M}=(M, f, F)$ and $\mathcal{N}=(N, g, G)$ are $\xi$-manifolds over $X$, their disjoint union defines a $\xi$-manifold $\mathcal{M}+\mathcal{N}=(M \sqcup N, f \sqcup g, F \sqcup G)$. The empty $\xi$-manifold will be denoted by $\mathcal{O}=(\emptyset, \emptyset, \emptyset)$.

We introduce a bordism relation on the set of $n$-dimensional $\xi$-manifolds $\mathcal{M}=$ $(M, f, F)$ over $X$, as follows. We say that $\mathcal{M} \sim \mathcal{O}$ if there exists triple $(W, \varphi, \Phi)$ consisting of a compact $(n+1)$-manifold $W$ with boundary $\partial W=M$, a continuous $\operatorname{map} \varphi: W \rightarrow X$ such that $\left.\varphi\right|_{\partial W}=f$, and a bundle isomorphism

$$
\Phi: T W \oplus \varphi^{*} \xi^{-} \oplus \varepsilon^{r} \stackrel{\simeq}{\longrightarrow} \varphi^{*} \xi^{+} \oplus \varepsilon^{s}
$$

whose restriction to $\partial W$ is equivalent to $F$ (here, we use the inward pointing normal vector to make the identification $\left.\left.T W\right|_{\partial W} \cong T M \oplus \varepsilon^{1}\right)$. Two $\xi$-manifolds $\mathcal{M}$ and $\mathcal{N}$ are bordant, written as $\mathcal{M} \sim \mathcal{N}$, if $\mathcal{M}-\mathcal{N} \sim \mathcal{O}$. Bordism is an equivalence relation, and the set of bordism classes of $n$-dimensional $\xi$-manifolds over $X$ is denoted by $\Omega_{n}(X ; \xi)$. A group structure on $\Omega_{n}(X ; \xi)$ is defined by setting

$$
[\mathcal{M}]+[\mathcal{N}]=[\mathcal{M}+\mathcal{N}], \quad-[\mathcal{M}]=[-\mathcal{M}], \quad 0=[\mathcal{O}]
$$

The resulting abelian group is called the $n$th normal bordism group of $X$ with coefficients in $\xi$.

The normal bordism groups are functorial with respect to morphisms of virtual bundles. Let $\bar{h}: \zeta \rightarrow \xi$ be a morphism of virtual bundles covering $h: Y \rightarrow X$, and let $\mathcal{M}=(M, f, F)$ be a $\zeta$-manifold over $Y$. Then the triple $h_{*} \mathcal{M}=(M, h \circ f, F)$ defines a $\xi$-manifold over $X$ (here, we use the canonical isomorphism $\zeta \cong h^{*} \xi$ ). This induces a homomorphism of abelian groups

$$
h_{*}: \Omega_{n}(Y ; \zeta) \rightarrow \Omega_{n}(X ; \xi), \quad h_{*}[\mathcal{M}]=\left[h_{*} \mathcal{M}\right]
$$

The normal bordism groups enjoy many properties analogous to the EilenbergSteenrod axioms for singular homology. Here, we recall a subset of these which will be needed in the sequel.

Dimension zero. There is an isomorphism

$$
\Omega_{0}(X ; \xi) \stackrel{\simeq}{\longrightarrow} H_{0}(X ; \mathbb{Z}(\xi))
$$

where $\mathbb{Z}(\xi)$ denotes the local system of integer coefficients twisted by $w_{1}(\xi)$. In particular, $\Omega_{0}(X ; \xi)$ is a direct sum over the path components of $X$ of groups isomorphic to $\mathbb{Z}$ or $\mathbb{Z}_{2}$, depending on whether the restriction of $\xi$ to the corresponding component is orientable or not. 
Homotopy invariance $I$. Let $H$ be a homotopy between maps $h_{0}, h_{1}: Y \rightarrow X$. Then the following diagram commutes:

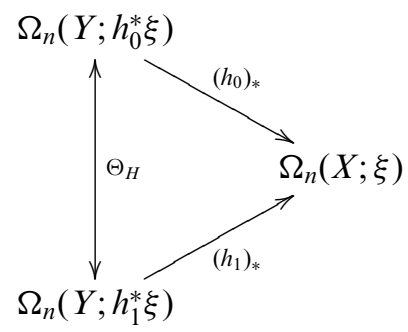

where the correspondence $\Theta_{H}$ is given by the isomorphism $h_{0}^{*} \xi \cong h_{1}^{*} \xi$ determined by $H$.

Homotopy invariance II. Let $h: Y \rightarrow X$ be a map such that

$$
h_{*}: \pi_{i}\left(Y, y_{0}\right) \rightarrow \pi_{i}\left(X, h\left(y_{0}\right)\right)
$$

is an isomorphism for $i \leq n$ and an epimorphism for $i=n+1$, with respect to any choice of base point $y_{0} \in Y$. Then the induced map

$$
h_{*}: \Omega_{i}\left(Y ; h^{*} \xi\right) \rightarrow \Omega_{i}(X ; \xi)
$$

is an isomorphism for $i \leq n$ and an epimorphism for $i=n+1$.

Gysin sequence. Let $v$ be an orthogonal vector bundle over $X$ of rank $k$, with associated sphere bundle $p: S v \rightarrow X$. There is a long exact sequence

$$
\cdots \rightarrow \Omega_{n}\left(S v ; p^{*} \xi\right) \stackrel{p_{*}}{\longrightarrow} \Omega_{n}(X ; \xi) \stackrel{e(v)}{\longrightarrow} \Omega_{n-k}(X ; \xi-v) \stackrel{w(v)}{\longrightarrow} \Omega_{n-1}(S v ; \xi) \rightarrow \cdots
$$

of normal bordism groups. The homomorphism $e(v)$ is called the Euler mapping.

We shall need to understand the Euler mapping in more detail. Let $[M, f, F] \in$ $\Omega_{n}(X ; \xi)$. Let $s: M \rightarrow E f^{*} v$ be a section transverse to the zero section $M \subseteq E f^{*} v$. Then the zeroes of $s$ form an $(n-k)$-dimensional submanifold $N \subseteq M$. Let $g=\left.f\right|_{N}: N \rightarrow$ $X$, then the normal bundle of $N$ in $M$ is isomorphic to $g^{*} v$, and restriction of $F$ to $N$ gives a bundle isomorphism

$$
G: T N \oplus g^{*} v \oplus g^{*} \xi^{-} \oplus \varepsilon^{r} \stackrel{\simeq}{\longrightarrow} g^{*} \xi^{+} \oplus \varepsilon^{s} .
$$

We then have $e(v)([M, f, F])=[N, g, G] \in \Omega_{n-k}(X ; \xi-v)$.

A particular case of interest to us is the Gysin sequence associated with a double cover. Let $\pi: \widetilde{X} \rightarrow X$ be a double cover, and let $\lambda$ be the associated line bundle over $X$, which has $S \lambda=\widetilde{X}$. Writing $\widetilde{\xi}$ for $\pi^{*} \xi$, we obtain a Gysin sequence

$$
\cdots \rightarrow \Omega_{n+1}(\widetilde{X} ; \widetilde{\xi}) \stackrel{\pi_{*}}{\longrightarrow} \Omega_{n+1}(X ; \xi) \stackrel{e(\lambda)}{\longrightarrow} \Omega_{n}(X ; \xi-\lambda) \stackrel{w(\lambda)}{\longrightarrow} \Omega_{n}(\widetilde{X} ; \widetilde{\xi}) \rightarrow \cdots
$$

where the map $w(\lambda)$ is induced by taking double covers. We refer the reader to [1, Chapter I.8] and [12, Section 10] for more details. 
We shall apply the Gysin sequence of a double cover to obtain information about the low-dimensional $\mathbb{Z}_{2}$-equivariant normal bordism groups of a space with involution. First, we recall some terminology and notation. Let $G$ be a group, and let $Y$ be a space on which $G$ acts. A $G$-vector bundle over $Y$ is a $G$-equivariant map $\xi \rightarrow Y$, which is a vector bundle in the usual sense, and such that each $\tau \in G$ induces a vector bundle map $\bar{\tau}: \xi \rightarrow \xi$. A virtual $G$-bundle over $Y$ is a formal difference $\xi=\xi^{+}-\xi^{-}$of $G$-vector bundles over $Y$.

The Borel space of $Y$ is the quotient $Y_{G}:=E G \times{ }_{G} Y$ of $E G \times Y$ by the diagonal $G$-action, where $E G$ is a contractible space on which $G$ acts freely. This construction is functorial; in particular, a $G$-vector bundle $\xi$ over $Y$ gives rise to a vector bundle $\xi_{G}$ over $Y_{G}$ of the same dimension. Similarly, given a virtual $G$-bundle $\xi=\xi^{+}-\xi^{-}$over $Y$, we get a virtual bundle $\xi_{G}=\xi_{G}^{+}-\xi_{G}^{-}$over $Y_{G}$. We then define the $n t h G$-equivariant normal bordism group of $Y$ with coefficients in $\xi$ to be the group $\Omega_{n}\left(Y_{G} ; \xi_{G}\right)$.

When $G=\mathbb{Z}_{2}$, the quotient map $\pi: E \mathbb{Z}_{2} \times Y \rightarrow Y_{\mathbb{Z}_{2}}$ is a double cover, and is homotopically equivalent to the $\operatorname{map} i: Y \rightarrow Y_{\mathbb{Z}_{2}}$ given by $i(y)=[e, y]$ for some choice of base point $e \in E \mathbb{Z}_{2}$.

Proposition 2.1. Let $\xi$ be a virtual $\mathbb{Z}_{2}$-bundle over a space with involution $t: Y \rightarrow Y$. Let $\Omega_{0}(Y ; \xi)$ have the $\mathbb{Z}_{2}$-module structure given by

$$
t_{*}: \Omega_{0}(Y ; \xi) \rightarrow \Omega_{0}(Y ; \xi)
$$

Then the map $i: Y \rightarrow Y_{\mathbb{Z}_{2}}$ induces an isomorphism of abelian groups

$$
i_{*}: H_{0}\left(\mathbb{Z}_{2} ; \Omega_{0}(Y ; \xi)\right)=\frac{\Omega_{0}(Y ; \xi)}{\left\{a-t_{*} a \mid a \in \Omega_{0}(Y ; \xi)\right\}} \stackrel{\simeq}{\longrightarrow} \Omega_{0}\left(Y_{\mathbb{Z}_{2}} ; \xi_{\mathbb{Z}_{2}}\right)
$$

Proof. The Gysin sequence for the double cover $Y \simeq E \mathbb{Z}_{2} \times Y \rightarrow Y_{\mathbb{Z}_{2}}$ ends

$$
\cdots \longrightarrow \Omega_{0}\left(Y_{\mathbb{Z}_{2}} ; \xi_{\mathbb{Z}_{2}}-\lambda\right) \stackrel{w(\lambda)}{\longrightarrow} \Omega_{0}(Y ; \xi) \stackrel{i_{*}}{\longrightarrow} \Omega_{0}\left(Y_{\mathbb{Z}_{2}} ; \xi_{\mathbb{Z}_{2}}\right) \longrightarrow 0 .
$$

It is not difficult to check that the image of $w(\lambda)$ is the subgroup

$$
\left\{a-t_{*} a \mid a \in \Omega_{0}(Y ; \xi)\right\} \subseteq \Omega_{0}(Y ; \xi)
$$

3. Hatcher-Quinn invariants. In this section, we recall some definitions and results of Hatcher and Quinn [4], who defined a regular homotopy invariant $\mu(f)$ which vanishes if (and in a certain dimension range, only if) the immersion $f$ is regularly homotopic to an embedding. We use the conventions for normal bordism groups set out in the previous section.

Let $f: N^{n} \rightarrow M^{m}$ be an immersion. The homotopy pullback

$$
P(f, f)=\left\{(x, \gamma, y) \in N \times M^{I} \times N \mid f(x)=\gamma(0) \text { and } f(y)=\gamma(1)\right\}
$$


fits into a homotopy commutative diagram

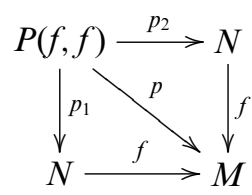

where $p_{1}(x, \gamma, y)=x, p_{2}(x, \gamma, y)=y$ and $p(x, \gamma, y)=\gamma(1 / 2)$. It has the following universal property: if $T$ is another space with maps $\rho_{1}, \rho_{2}: T \rightarrow N$ such that $f \rho_{1} \simeq f \rho_{2}$, then there is a map $\phi: T \rightarrow P(f, f)$, unique up to homotopy, such that $p_{1} \phi \simeq \rho_{1}$ and $p_{2} \phi \simeq \rho_{2}$.

Now suppose that $f: N^{n} \rightarrow M^{m}$ is self-transverse, and $N$ is closed. Then the space

$$
\bar{\Sigma}(f)=\{(x, y) \in N \times N \mid f(x)=f(y) \text { and } x \neq y\}
$$

is a closed submanifold of $N \times N$ of dimension $2 n-m$, the so-called self-intersection manifold of $f$. The projections $\rho_{1}, \rho_{2}: \bar{\Sigma}(f) \rightarrow N$ ensure that there is a homotopy commutative diagram

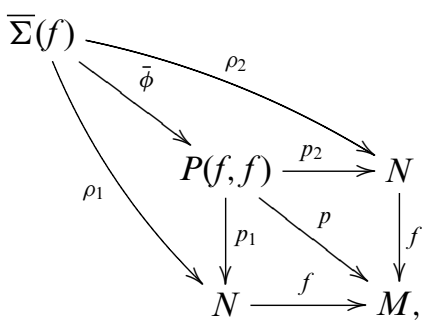

where $\bar{\phi}(x, y)=\left(x, c_{f(x)}, y\right)$ for $c_{f(x)}$ the constant path at $f(x)$.

Let $\psi: \bar{\Sigma}(f) \rightarrow M$ be the composition $p \bar{\phi}$, so $\psi(x, y)=f(x)=f(y)$. The selfintersection manifold fits into a pullback diagram

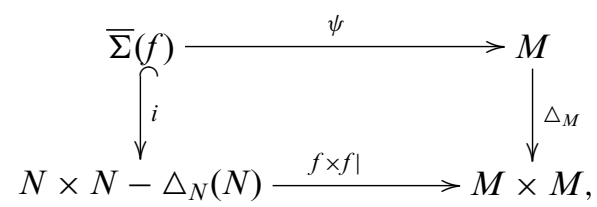

where for a space $X$ we denote by $\triangle_{X}: X \rightarrow X \times X$ the diagonal map $x \mapsto(x, x)$. The embedding $i: \bar{\Sigma}(f) \hookrightarrow N \times N$ factors as $\left(\rho_{1} \times \rho_{2}\right) \triangle_{\bar{\Sigma}(f)}$. We therefore have a sequence of vector bundle isomorphisms

$$
\begin{aligned}
T \bar{\Sigma}(f) \oplus \psi^{*} T M & \cong T \bar{\Sigma}(f) \oplus \psi^{*} v_{\triangle_{M}} \\
& \cong T \bar{\Sigma}(f) \oplus v_{i} \\
& \cong i^{*} T(N \times N-\triangle N) \\
& \cong \rho_{1}^{*} T N \oplus \rho_{2}^{*} T N,
\end{aligned}
$$


where $v$ denotes a normal bundle. Now note that each of the maps $\psi, \rho_{1}$ and $\rho_{2}$ factor through $\bar{\phi}: \bar{\Sigma}(f) \rightarrow P(f, f)$, and so we have constructed a bundle isomorphism

$$
\bar{\Phi}: T \bar{\Sigma}(f) \oplus \bar{\phi}^{*} p^{*} T M \stackrel{\simeq}{\longrightarrow} \bar{\phi}^{*}\left(p_{1}^{*} T N \oplus p_{2}^{*} T N\right) .
$$

It follows that the self-intersection manifold of $f$ represents an element

$$
[\bar{\Sigma}(f), \bar{\phi}, \bar{\Phi}] \in \Omega_{2 n-m}(P(f, f) ; \zeta), \quad \zeta=p_{1}^{*} T N \oplus p_{2}^{*} T N-p^{*} T M
$$

In order that we do not count each double point twice, however, we must factor out by the action of the cyclic group $\mathbb{Z}_{2}$, which acts on all the manifolds in diagram (20) by swapping factors. In particular, $\mathbb{Z}_{2}$ acts freely on $\bar{\Sigma}(f)$, with quotient

$$
\Sigma(f)=\bar{\Sigma}(f) / \mathbb{Z}_{2}=\{[x, y] \mid(x, y) \in \bar{\Sigma}(f)\},
$$

the so-called double-point manifold of $f$. Let $e: \bar{\Sigma}(f) \rightarrow E \mathbb{Z}_{2}=S^{\infty}$ classify the double cover $\pi: \bar{\Sigma}(f) \rightarrow \Sigma(f)$, and define a map

$$
\phi: \Sigma(f) \rightarrow P(f, f)_{\mathbb{Z}_{2}}, \quad \phi[x, y]=[e(x, y), \bar{\phi}(x, y)]=\left[e(x, y),\left(x, c_{f(x)}, y\right)\right] .
$$

There is an involution

$$
t: P(f, f) \rightarrow P(f, f), \quad t(x, \gamma, y)=(y, \bar{\gamma}, x), \quad \bar{\gamma}(t)=\gamma(1-t),
$$

which is covered by the bundle involutions

$$
\begin{array}{rlrl}
\bar{t}: p^{*} T M & \rightarrow p^{*} T M, & \bar{t}(v) & =-v, \\
\bar{t}: p_{1}^{*} T N \oplus p_{2}^{*} T N & \rightarrow p_{1}^{*} T N \oplus p_{2}^{*} T N, \bar{t}\left(v_{1}, v_{2}\right)=\left(v_{2}, v_{1}\right) .
\end{array}
$$

Factoring out by the $\mathbb{Z}_{2}$-action, we find that $\bar{\Phi}$ induces a stable bundle isomorphism

$$
\Phi: T \Sigma(f) \oplus \phi^{*}\left(p^{*} T M\right)_{\mathbb{Z}_{2}} \stackrel{\simeq}{\longrightarrow} \phi^{*}\left(p_{1}^{*} T N \oplus p_{1}^{*} T N\right)_{\mathbb{Z}_{2}} .
$$

Definition 3.1 Hatcher-Quinn [4]. Let $f: N^{n} \rightarrow M^{m}$ be a self-transverse immersion with $N$ closed. The Hatcher-Quinn invariant of $f$ is the normal bordism class

$$
\mu(f)=[\Sigma(f), \phi, \Phi] \in \Omega_{2 n-m}\left(P(f, f)_{\mathbb{Z}_{2}} ; \zeta_{\mathbb{Z}_{2}}\right),
$$

where $\zeta_{\mathbb{Z}_{2}}$ is the virtual vector bundle $\left(p_{1}^{*} T N \oplus p_{2}^{*} T N\right)_{\mathbb{Z}_{2}}-\left(p^{*} T M\right)_{\mathbb{Z}_{2}}$.

REMARK 3.1. If $f$ is not self-transverse, then we define $\mu(f)=\mu\left(f^{\prime}\right)$, where $f^{\prime}: N \hookrightarrow$ $M$ is a self-transverse immersion regularly homotopic to $f$. This is well-defined by the following result.

THEOREM 3.3 (Hatcher-Quinn [4, Theorem 2.3]). The class $\mu(f)$ is a regular homotopy invariant. If $2 m \geq 3(n+1)$ and $\mu(f)=[\mathcal{N}]$ for some singular $\zeta_{\mathbb{Z}_{2}}$-manifold $\mathcal{N}=\left(N^{2 n-m}, \gamma, \Gamma\right)$ in $P(f, f)_{\mathbb{Z}_{2}}$, then $f$ is regularly homotopic to an immersion $g$ with $\Sigma(g)=N$. In particular, $\mu(f)=0$ if and only iff is regularly homotopic to an embedding.

For an alternative approach to this result, see the papers of Klein and Williams $[6,7]$ on homotopical intersection theory. 
4. Wall's invariant. In order to investigate the possibility of performing surgery in the middle dimension on non-simply-connected manifolds, Wall [15] defined an obstruction to a given immersion $f: S^{n} \rightarrow M^{2 n}$ being regularly homotopic to an embedding. Wall's obstruction is complete when $n \geq 3$. In this section, we briefly recall the construction, following [15] (see also [16, Chapter 5]).

Wall's invariant $\mu_{W}(f)$ for a self-transverse immersion $f: N^{n} \rightarrow M^{2 n}$, where $N$ is closed and simply-connected and $M$ is connected, may be described as follows. Choose once and for all a base point $n_{0} \in N$ for which $f^{-1}\left(\left\{f\left(n_{0}\right)\right\}\right)=\left\{n_{0}\right\}$, and let $m_{0}=f\left(n_{0}\right)$ be the base point of $M$. Wall's obstruction lives in a quotient of the integral group ring $\mathbb{Z}[\pi]$ of $\pi=\pi_{1}\left(M, m_{0}\right)$. In particular, if $w: \pi \rightarrow\{ \pm 1\}$ is the orientation character of $M$, then we may define an involution on the group ring,

$$
\overline{()}: \mathbb{Z}[\pi] \rightarrow \mathbb{Z}[\pi], \quad \sum_{\sigma \in \pi} n_{\sigma} \sigma \mapsto \sum_{\sigma \in \pi}(-1)^{n} w(\sigma) n_{\sigma} \sigma^{-1} .
$$

This makes $\mathbb{Z}[\pi]$ a $\mathbb{Z}_{2}$-module, and $\mu_{W}(f)$ will be an element of the group of coinvariants

$$
H_{0}\left(\mathbb{Z}_{2} ; \mathbb{Z}[\pi]\right)=\frac{\mathbb{Z}[\pi]}{\{a-\bar{a} \mid a \in \mathbb{Z}[\pi]\}}
$$

The above conditions on $f: N \leftrightarrow M$ ensure that the self-intersection $\bar{\Sigma}(f)$ and the double-point manifold $\Sigma(f)$ each consist of a finite number of points. Each double point $[x, y] \in \Sigma(f)$ may be lifted to a self-intersection $(x, y) \in \bar{\Sigma}(f)$ by an arbitrary choice of ordering. For each self-intersection, we define an element $\sigma_{(x, y)} \in \pi$ and a $\operatorname{sign} \varepsilon_{(x, y)} \in\{ \pm 1\}$ as follows. Choose paths $\gamma_{x}, \gamma_{y}$ in $N$ from $n_{0}$ to $x$ and $y$, respectively, which avoid other self-intersection points of $f$. Then $\sigma_{(x, y)} \in \pi$ is defined to be the homotopy class of the loop $f \gamma_{x} \cdot f \overline{\gamma_{y}}$ in $M$ based at $m_{0}$. Note that changing the order of $x$ and $y$ reverses the loop, so $\sigma_{(y, x)}=\sigma_{(x, y)}{ }^{-1}$. To define the sign, fix orientations of $N$ at $n_{0}$ and $M$ at $m_{0}$. The tangent spaces $T N_{x}$ and $T N_{y}$ become oriented by transport along $\gamma_{x}$ and $\gamma_{y}$ of the orientation of $T N_{n_{0}}$. Set $\varepsilon_{(x, y)}$ to equal 1 if the orientation of $d f\left(T N_{x}\right)$ followed by that of $d f\left(T N_{y}\right)$ agrees with the transport of the orientation of $T M_{m_{0}}$ along $f \gamma_{x}$, and equal to -1 otherwise. Note that $\varepsilon_{(y, x)}=(-1)^{n} w\left(\sigma_{(x, y)}\right) \varepsilon_{(x, y)}$.

Definition 4.1. Let $f: N^{n} \rightarrow M^{2 n}$ be a self-transverse immersion, with $N$ closed and simply-connected and $M$ connected. The Wall invariant of $f$ is the well-defined class $\mu_{W}(f) \in H_{0}\left(\mathbb{Z}_{2} ; \mathbb{Z}[\pi]\right)$ represented by the finite sum

$$
\tilde{\mu}_{W}(f)=\sum_{[x, y] \in \Sigma(f)} \varepsilon_{(x, y)} \sigma_{(x, y)} \in \mathbb{Z}[\pi] .
$$

THEOREM 4.2 Wall [15, Theorem 3.1]. The class $\mu_{W}(f)$ is a regular homotopy invariant. If $f$ is regularly homotopic to an embedding, then $\mu_{W}(f)=0$. Conversely, if $n \geq 3$ and $\mu_{W}(f)=0$, then $f$ is regularly homotopic to an embedding.

5. Proof of Theorem A. In this section, we prove that the Hatcher-Quinn and Wall invariants of a self-transverse immersion $f: N^{n} \rightarrow M^{2 n}$, where $N$ is closed and 
simply-connected and $M$ is connected, reside in isomorphic groups and correspond under this isomorphism.

Let $\mathbb{Z}[\pi]$ be the $\mathbb{Z}_{2}$-module described in Section 4 , where $\pi=\pi_{1}\left(M, m_{0}\right)$. Recall that the normal bordism group $\Omega_{0}(P(f, f) ; \zeta)$ (see Section 3 ) also has the structure of a $\mathbb{Z}_{2}$-module, given by the involution

$$
t_{*}: \Omega_{0}(P(f, f) ; \zeta) \rightarrow \Omega_{0}(P(f, f) ; \zeta)
$$

where $t: P(f, f) \rightarrow P(f, f)$ is the involution $t(x, \gamma, y)=(y, \bar{\gamma}, x)$.

LEMMA 5.1. There is an isomorphism of $\mathbb{Z}_{2}$-modules

$$
\chi: \mathbb{Z}[\pi] \stackrel{\simeq}{\longrightarrow} \Omega_{0}(P(f, f) ; \zeta) .
$$

Proof. Consider the fibration $\left(p_{1}, p_{2}\right): P(f, f) \rightarrow N \times N$ with fibre $\Lambda M=$ $\Lambda\left(M, m_{0}\right)$ the based loop space of $M$. Since $N$ is simply-connected, the fibre inclusion

$$
\iota: \Lambda M \rightarrow P(f, f), \quad \iota(\gamma)=\left(n_{0}, \gamma, n_{0}\right)
$$

induces an isomorphism $\iota_{*}: \pi_{0}(\Lambda M) \stackrel{\simeq}{\longrightarrow} \pi_{0}(P(f, f))$, and hence induces an isomorphism

$$
\iota_{*}: \Omega_{0}\left(\Lambda M ; \iota^{*} \zeta\right) \stackrel{\simeq}{\longrightarrow} \Omega_{0}(P(f, f) ; \zeta)
$$

Now $\iota^{*} \zeta=c^{*} T N \oplus c^{*} T N-\operatorname{ev}^{*} T M$, where $c: \Lambda M \rightarrow N$ is a constant at $n_{0}$ and the evaluation map ev: $\Lambda M \rightarrow M$ given by $\operatorname{ev}(\gamma)=\gamma(1 / 2)$ is null-homotopic via the homotopy $\gamma \mapsto \gamma((1-t) 1 / 2)$. Hence, $\iota^{*} \zeta$ is a trivial virtual bundle, and in particular, is orientable over each path component $\Lambda M_{\sigma} \subseteq \Lambda M$. Thus, there are isomorphisms of abelian groups

$$
\mathbb{Z}[\pi] \cong \Omega_{0}\left(\Lambda M ; \iota^{*} \zeta\right) \cong \Omega_{0}(P(f, f) ; \zeta)
$$

We give an explicit isomorphism $\chi: \mathbb{Z}[\pi] \stackrel{\simeq}{\longrightarrow} \Omega_{0}(P(f, f) ; \zeta)$ by choosing a generator $\chi(\sigma) \in \Omega_{0}(P(f, f) ; \zeta)$ for each $\sigma \in \pi$, and show that $\chi$ is a map of $\mathbb{Z}_{2}$-modules.

Let $\gamma$ be a loop in $M$ representing $\sigma$. Fix orientations for the tangent spaces $T N_{n_{0}}$ and $T M_{m_{0}}$. These induce orientations of $T M_{\gamma(1 / 2)}$ by parallel transport along the first half of $\gamma$, and of $T N_{n_{0}} \oplus T N_{n_{0}}$ by direct sum. We then set

$$
\chi(\sigma)=\left[P^{0},\left(n_{0}, \gamma, n_{0}\right), \Xi\right], \quad \Xi: T M_{\gamma(1 / 2)} \stackrel{\simeq}{\longrightarrow} T N_{n_{0}} \oplus T N_{n_{0}},
$$

where $P^{0}$ is a point and $\Xi$ is orientation preserving. It is easy to see that $\chi(\sigma) \in$ $\Omega_{0}(P(f, f) ; \zeta)$ does not depend on the choices of $\gamma$ and $\Xi$.

Let $t: P(f, f) \rightarrow P(f, f)$ be the involution. In order to show that $\chi$ is a $\mathbb{Z}_{2}$-module map, we must show that $t_{*} \chi(\sigma)=(-1)^{n} w(\sigma) \chi\left(\sigma^{-1}\right)$. Now $t_{*} \chi(\sigma)=\left[P,\left(n_{0}, \bar{\gamma}, n_{0}\right), \Psi\right]$, where $\Psi$ is the vector space isomorphism determined by the diagram

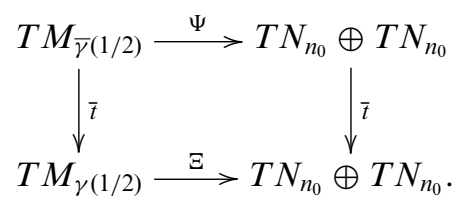


Orient $T M_{\bar{\gamma}(1 / 2)}$ by parallel transport along the first half of $\bar{\gamma}$. We wish to determine the sign of the linear map $\Psi$. If $T M_{\gamma(1 / 2)}$ is oriented by transport along the first half of $\gamma$, then the linear map $\bar{t}=(-1): T M_{\bar{\gamma}(1 / 2)} \rightarrow T M_{\gamma(1 / 2)}$ has $\operatorname{sign}(-1)^{2 n} w(\sigma)=w(\sigma)$. The map $\Xi$ has sign +1 . The map $\bar{t}: T N_{n_{0}} \oplus T N_{n_{0}} \rightarrow T N_{n_{0}} \oplus T N_{n_{0}}$ which swaps factors has sign $(-1)^{n^{2}}=(-1)^{n}$. Thus, $\Psi$ has sign $(-1)^{n} w(\sigma)$, and $t_{*} \chi(\sigma)=(-1)^{n} w(\sigma) \chi\left(\sigma^{-1}\right)$ as claimed.

Combining this lemma with Proposition 2.1, we have group isomorphisms

$$
H_{0}\left(\mathbb{Z}_{2} ; \mathbb{Z}[\pi]\right) \stackrel{\chi_{*}}{\longrightarrow} H_{0}\left(\mathbb{Z}_{2} ; \Omega_{0}(P(f, f) ; \zeta)\right) \stackrel{i_{*}}{\longrightarrow} \Omega_{0}\left(P(f, f)_{\mathbb{Z}_{2}} ; \zeta_{\mathbb{Z}_{2}}\right)
$$

where $i: P(f, f) \rightarrow P(f, f)_{\mathbb{Z}_{2}}$ is given by $i(x, \gamma, y)=[e,(x, \gamma, y)]$ for some base point $e \in E \mathbb{Z}_{2}$. Set $\mathcal{F}=i_{*} \circ \chi_{*}$. The proof of Theorem $\mathrm{A}$ is completed by the following lemma:

LEMMA 5.2. $\mathcal{F}\left(\mu_{W}(f)\right)=i_{*}\left[\chi \tilde{\mu}_{W}(f)\right]=\mu(f)$.

Proof. For each double point $[x, y] \in \Sigma(f)$, we choose a lift $(x, y) \in \bar{\Sigma}(f)$ and paths $\gamma_{x}$ and $\gamma_{y}$ in $N$ from $n_{0}$ to $x$ and $y$, respectively. Then

$$
\chi \tilde{\mu}_{W}(f)=[\Sigma(f), \psi, \Upsilon] \in \Omega_{0}(P(f, f) ; \zeta)=\Omega_{0}\left(P(f, f) ; i^{*} \zeta_{\mathbb{Z}_{2}}\right),
$$

where $\psi[x, y]=\left(n_{0}, f \gamma_{x} \cdot f \overline{\gamma_{y}}, n_{0}\right)$ and over $[x, y] \in \Sigma(f)$, the stable isomorphism

$$
\Upsilon: T M_{f(x)} \stackrel{\simeq}{\longrightarrow} T N_{n_{0}} \oplus T N_{n_{0}}
$$

has $\operatorname{sign} \varepsilon_{(x, y)}($ see Section 4). So,

$$
i_{*} \chi_{*} \mu_{W}(f)=[\Sigma(f), i \circ \psi, \Upsilon] \in \Omega_{0}\left(P(f, f)_{\mathbb{Z}_{2}} ; \zeta_{\mathbb{Z}_{2}}\right) .
$$

We next observe that the maps $i \circ \psi, \phi: \Sigma(f) \rightarrow P(f, f)_{\mathbb{Z}_{2}}$, given by

$$
i \circ \psi[x, y]=\left[e,\left(n_{0}, f \gamma_{x} \cdot f \bar{\gamma}_{y}, n_{0}\right)\right], \quad \phi[x, y]=\left[e(x, y),\left(x, c_{f(x)}, y\right)\right],
$$

are homotopic. For each $[x, y] \in \Sigma(f)$, choose a path $\omega_{(x, y)}: I \rightarrow E \mathbb{Z}_{2}$ from $e$ to $e(x, y)$. For any path $\gamma: I \rightarrow N$ and $t \in I$, define a re-parameterised path $\gamma^{t}$ (whose image is $\gamma([t, 1]))$ by setting $\gamma^{t}(s)=\gamma((1-t) s+t)$. Now the desired homotopy $H: \Sigma(f) \times I \rightarrow$ $P(f, f)_{\mathbb{Z}_{2}}$ is defined by

$$
H([x, y], t)=\left[\omega_{(x, y)}(t),\left(\gamma_{x}(t), f \gamma_{x}^{t} \cdot f \overline{\gamma_{y}^{t}}, \gamma_{y}(t)\right)\right] .
$$

By the first property of homotopy invariance of the bordism groups in Section 2, to complete the proof, it suffices to check that for each double point $[x, y] \in \Sigma(f)$, the diagram of vector space isomorphisms

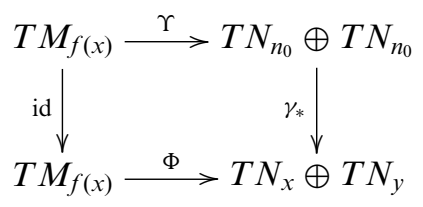


commutes up to sign. Here, the vertical isomorphisms are those induced by the homotopy $H$, and $\gamma_{*}$ stands for parallel transport along $\gamma_{x}$ on the first summand and $\gamma_{y}$ on the second. The isomorphism $\Phi$ is described in Section 3, and may be seen to have inverse given by $\left(v_{1}, v_{2}\right) \mapsto d f_{x}\left(v_{1}\right)-d f_{y}\left(v_{2}\right)$. The diagram commutes up to sign by the definition of $\varepsilon_{(x, y)}$. This completes the proof of the lemma and Theorem A.

6. The case $f: N^{n} \rightarrow M^{2 n-1}$. In this final section, we offer some speculative remarks concerning the case of an immersion $f: N^{n} \rightarrow M^{2 n-1}$, where $N$ is closed and simply-connected and $M$ is connected.

In this case, if $n \geq 5$, then $f$ is regularly homotopic to an embedding if and only if the Hatcher-Quinn invariant

$$
\mu(f) \in \Omega_{1}\left(P(f, f)_{\mathbb{Z}_{2}} ; \zeta_{\mathbb{Z}_{2}}\right)
$$

vanishes. We propose to investigate the vanishing of $\mu(f)$ using the Gysin sequence of the double cover $P(f, f) \simeq E \mathbb{Z}_{2} \times P(f, f) \rightarrow P(f, f)_{\mathbb{Z}_{2}}$ (see Section 2). We abbreviate $P=P(f, f)$, and look at the portion of this sequence

$$
\cdots \longrightarrow \Omega_{1}(P ; \zeta) \stackrel{i_{*}}{\longrightarrow} \Omega_{1}\left(P_{\mathbb{Z}_{2}} ; \zeta_{\mathbb{Z}_{2}}\right) \stackrel{e}{\longrightarrow} \Omega_{0}\left(P_{\mathbb{Z}_{2}} ; \zeta_{\mathbb{Z}_{2}}-\lambda\right) \longrightarrow \cdots
$$

Here, $\lambda$ is the line bundle associated wity the double cover $E \mathbb{Z}_{2} \times P \rightarrow P_{\mathbb{Z}_{2}}$, and $e$ is the Euler mapping.

Proposition 6.1. Let $f$ : $N^{n} \rightarrow M^{2 n-1}$ be a self-transverse immersion, where $N$ is closed and simply-connected, $M$ is connected and $n \geq 3$. Consider the relaxed immersion

$$
g=(f, 0): N \rightarrow M \times \mathbb{R}, \quad g(n)=(f(n), 0) .
$$

Then $e(\mu(f))=0$ if and only if $\mu(g)=0$ if and only if $g$ is regularly homotopic to an embedding.

Proof. Let $h: N \rightarrow \mathbb{R}$ be a smooth function such that the immersion

$$
g^{\prime}: N \rightarrow M \times \mathbb{R}, \quad g^{\prime}(n)=(f(n), h(n))
$$

is self-transverse. Note that $g^{\prime}$ is regularly homotopic to $g$, and so $\mu\left(g^{\prime}\right)=\mu(g)$. The anti-symmetric mapping

$$
\bar{\varphi}: \bar{\Sigma}(f) \rightarrow \mathbb{R}, \quad \bar{\varphi}(x, y)=h(x)-h(y)
$$

defines a section $\varphi: \Sigma(f) \rightarrow \bar{\Sigma}(f) \times_{\mathbb{Z}_{2}} \mathbb{R}$ of the line bundle $\lambda_{\pi}$ associated with the double cover $\pi: \bar{\Sigma}(f) \rightarrow \Sigma(f)$. The self-transversality of $g^{\prime}$ implies that $\varphi$ is transverse to the zero section, and the zeroes of $\varphi$ are exactly the double points $\Sigma\left(g^{\prime}\right) \subseteq \Sigma(f)$ of $g^{\prime}$.

Recall that $\mu(f)=[\Sigma(f), \phi, \Phi]$. The line bundle $\lambda_{\pi}$ can be identified with the pullback $\phi^{*} \lambda$. It follows from the description of the Euler mapping in Section 2 that

$$
e(\mu(f))=\left[\Sigma\left(g^{\prime}\right),\left.\phi\right|_{\Sigma\left(g^{\prime}\right)},\left.\Phi\right|_{\Sigma\left(g^{\prime}\right)}\right] \in \Omega_{0}\left(P_{\mathbb{Z}_{2}} ; \zeta_{\mathbb{Z}_{2}}-\lambda\right) .
$$


We now investigate $\mu\left(g^{\prime}\right)$. The homotopy pullback $P^{\prime}=P\left(g^{\prime}, g^{\prime}\right)$ comes with maps $p_{1}^{\prime}, p_{2}^{\prime}: P^{\prime} \rightarrow N, p^{\prime}: P^{\prime} \rightarrow M \times \mathbb{R}$, and

$$
\mu\left(g^{\prime}\right) \in \Omega_{0}\left(P_{\mathbb{Z}_{2}}^{\prime} ; \zeta_{\mathbb{Z}_{2}}^{\prime}\right), \quad \text { where } \quad \zeta^{\prime}=p_{1}^{\prime *} T N \oplus p_{2}^{\prime *} T N-p^{* *} T(M \times \mathbb{R}) .
$$

There is a canonical $\mathbb{Z}_{2}$-equivariant homotopy equivalence $\Pi: P^{\prime} \rightarrow P$, which projects a path in $M \times \mathbb{R}$ onto a path $M$, such that

$$
p_{1} \Pi=p_{1}^{\prime}, \quad p_{2} \Pi=p_{2}^{\prime} \quad \text { and } \quad p \Pi=\operatorname{pr} p^{\prime}, \quad \text { where pr: } M \times \mathbb{R} \rightarrow M .
$$

Let $\varepsilon_{P}^{-}$be the trivial line bundle over $P$ with $\mathbb{Z}_{2}$-action $((x, \gamma, y), v) \mapsto((y, \bar{\gamma}, x),-v)$, and note that $\lambda=\left(\varepsilon_{P}^{-}\right)_{\mathbb{Z}_{2}}$. It follows that $\Pi^{*}\left(\zeta_{\mathbb{Z}_{2}}-\lambda\right) \cong \zeta_{\mathbb{Z}_{2}}^{\prime}$, and $\Pi$ induces an isomorphism

$$
\Pi_{*}: \Omega_{0}\left(P_{\mathbb{Z}_{2}}^{\prime} ; \zeta_{\mathbb{Z}_{2}}^{\prime}\right) \stackrel{\simeq}{\longrightarrow} \Omega_{0}\left(P_{\mathbb{Z}_{2}} ; \zeta_{\mathbb{Z}_{2}}-\lambda\right)
$$

We claim that $e(\mu(f))=\Pi_{*} \mu\left(g^{\prime}\right)$, and hence $e(\mu(f))$ vanishes if and only if $\mu\left(g^{\prime}\right)=\mu(g)$ vanishes. By definition,

$$
\mu\left(g^{\prime}\right)=\left[\Sigma\left(g^{\prime}\right), \phi^{\prime}, \Phi^{\prime}\right] \in \Omega_{0}\left(P_{\mathbb{Z}_{2}} ; \zeta_{\mathbb{Z}_{2}}-\lambda\right),
$$

where $\phi^{\prime}(x, y)=\left[e(x, y),\left(x, c_{g^{\prime}(x)}, y\right)\right]$ and

$$
\Phi^{\prime}: T \Sigma\left(g^{\prime}\right) \oplus \phi^{\prime *}\left(p^{\prime *} T(M \times \mathbb{R})\right)_{\mathbb{Z}_{2}} \stackrel{\simeq}{\longrightarrow} \phi^{\prime *}\left(p_{1}^{\prime *} T N \oplus p_{2}^{\prime *} T N\right)_{\mathbb{Z}_{2}} .
$$

The claim can be proved by noting that $\Pi \phi^{\prime}=\left.\phi\right|_{\Sigma\left(g^{\prime}\right)}$, and making the identifications

$$
\begin{gathered}
p^{\prime *} T(M \times \mathbb{R}) \cong p^{\prime *} \operatorname{pr}^{*}\left(T M \oplus \varepsilon_{M}^{-}\right) \cong \Pi^{*}\left(p^{*} T M \oplus \varepsilon_{P}^{-}\right), \\
p_{1}^{\prime *} T N \oplus p_{2}^{\prime *} T N=\Pi^{*}\left(p_{1}^{*} T N \oplus p_{2}^{*} T N\right),
\end{gathered}
$$

and noting that both $\left.\Phi\right|_{\Sigma\left(g^{\prime}\right)}$ and $\Phi^{\prime}$ arise from consideration of the embedding $i^{\prime}: \bar{\Sigma}\left(g^{\prime}\right) \hookrightarrow N \times N$.

The question of when the relaxed immersion $g: N \rightarrow M \times \mathbb{R}$ is regularly homotopic to an embedding has been considered by Wall [16, p. 83] and by Szücs $[14$, p. 252], and turns out to depend on the nature of the double circles of the original immersion $f$.

Recall that the construction of Wall's invariant requires choosing an ordering of each double point. In the case of $f: N^{n} \rightarrow M^{2 n-1}$, the double points are replaced by finitely many double circles $C \subseteq M$. Each double circle $C$ is doubly covered by its pre-image $\bar{C} \subseteq N$. Let us call a double circle $C$ trivial if the corresponding cover $\pi_{C}: \bar{C} \rightarrow C$ is trivial, and non-trivial otherwise. An ordering of the double points now corresponds to a section of $\pi_{C}$ over each trivial double circle. As the next proposition shows, non-trivial double circles give a first obstruction to $f$ being regularly homotopic to an embedding.

Proposition 6.2. Let $f: N^{n} \rightarrow M^{2 n-1}$ be as in the statement of Proposition 6.1. Then the relaxed immersion

$$
g=(f, 0): N \uparrow M \times \mathbb{R}, \quad g(n)=(f(n), 0)
$$


is regularly homotopic to an embedding if and only if the number of non-trivial double circles off in each one-dimensional homotopy class of $M$ is even.

Proof. As in the proof of Proposition 6.1, choose a smooth function $h: N \rightarrow$ $\mathbb{R}$ such that $g^{\prime}=(f, h): N \rightarrow M \times \mathbb{R}$ is self-transverse, and note that any such $g^{\prime}$ is regularly homotopic to $g$. Hence, $g$ is regularly homotopic to an embedding if and only if $0=\mu_{W}\left(g^{\prime}\right) \in H_{0}\left(\mathbb{Z}_{2} ; \mathbb{Z}\left[\pi_{1}(M \times \mathbb{R})\right]\right)=H_{0}\left(\mathbb{Z}_{2} ; \mathbb{Z}[\pi]\right)$, by Theorem 4.2. The rest of the proof consists of an analysis of when the non-simply-connected Whitney trick can be applied to the relaxed immersion $g^{\prime}$.

The double points of $g^{\prime}$ coincide with the zeroes of the section of $\lambda_{\pi}$ induced by the anti-symmetric mapping $\bar{\varphi}: \bar{\Sigma}(f) \rightarrow \mathbb{R}$ (see (49)). We may assume that $h$ was chosen to separate the two components of $\bar{C}$ for each trivial double circle $C \subseteq \Sigma(f)$. It follows that all the double points of $g^{\prime}$ lie on non-trivial double circles, and the number of double points on each circle is odd.

Let $[x, y]$ be a double point of $g^{\prime}$ lying on a non-trivial double circle $C$ of $f$. Then $\sigma_{(x, y)} \in \pi_{1}\left(M, m_{0}\right)$ is the homotopy class of a path that travels from $m_{0}$ to $C$ along the image under $f$ of a path in $N$, then around $C$, and then back to $m_{0}$ along the same path. Clearly, $\sigma_{(y, x)}=\sigma_{(x, y)}^{-1}=\sigma_{(x, y)}$ (since $C=f(\bar{C})$ and $N$ is simply-connected).

We now apply Wall's formula

$$
\lambda\left(g^{\prime}, g^{\prime}\right)=\tilde{\mu}_{W}\left(g^{\prime}\right)+\overline{\tilde{\mu}_{W}\left(g^{\prime}\right)}+\chi\left(g^{\prime}\right) \in \mathbb{Z}[\pi],
$$

where $\lambda\left(g^{\prime}, g^{\prime}\right) \in \mathbb{Z}[\pi]$ is the (non-simply-connected) intersection number of $g^{\prime}$ with a transverse approximation of $g^{\prime}$, and $\chi\left(g^{\prime}\right)$ denotes the Euler number of $v_{g^{\prime}}$ (see [16, Theorem 5.2]). However, $\lambda\left(g^{\prime}, g^{\prime}\right)=0$ (since $N$ is compact, the $\mathbb{R}$ coordinate in $M \times \mathbb{R}$ allows us to separate the two copies of $g^{\prime}$ ) and $\chi\left(g^{\prime}\right)=0$ (since $\left.v_{g^{\prime}} \cong v_{f} \oplus \varepsilon^{1}\right)$. Therefore

$$
0=\sum_{[x, y] \in \Sigma\left(g^{\prime}\right)}\left(\varepsilon_{(x, y)}+\varepsilon_{(y, x)}\right) \sigma_{(x, y)} .
$$

Let $[x, y] \in \Sigma\left(g^{\prime}\right)$ with $\varepsilon_{(x, y)}=\varepsilon_{(y, x)}$. Then there must be another double point $\left[x^{\prime}, y^{\prime}\right] \in \Sigma\left(g^{\prime}\right)$ with $\varepsilon_{\left(x^{\prime}, y^{\prime}\right)}=\varepsilon_{\left(y^{\prime}, x^{\prime}\right)}=-\varepsilon_{(x, y)}$ and $\sigma_{\left(x^{\prime}, y^{\prime}\right)}=\sigma_{(x, y)}$ to cancel it. In this case, these two double points contribute 0 to $\tilde{\mu}_{W}\left(g^{\prime}\right)$, so may be ignored. The remaining double points have $\varepsilon_{(x, y)}=-\varepsilon_{(y, x)}=-(-1)^{n} w\left(\sigma_{(x, y)}\right) \varepsilon_{(x, y)}$, and consequently $2\left[\sigma_{(x, y)}\right]=0 \in H_{0}\left(\mathbb{Z}_{2} ; \mathbb{Z}[\pi]\right)$. Hence,

$$
\mu_{W}\left(g^{\prime}\right)=\left[\sum_{[x, y] \in \Sigma\left(g^{\prime}\right)} \sigma_{(x, y)}\right]
$$

is zero if and only if the number of double points in each homotopy class is even. Since the number of double points on each non-trivial double circle is odd, the proposition follows.

Now if $n \geq 3$ and $f: N^{n} \rightarrow M^{2 n-1}$ is an immersion such that $g: N \rightarrow M \times \mathbb{R}$ is regularly homotopic to an embedding, then the sequence (46) tells us that the Hatcher-Quinn invariant $\mu(f)$ lifts non-uniquely to an element

$$
\bar{\mu}(f) \in \Omega_{1}(P(f, f) ; \zeta) .
$$

The vanishing of $\bar{\mu}(f)$ is a sufficient condition for $f$ to be regularly homotopic to an immersion. The next result identifies the group $\Omega_{1}(P(f, f) ; \zeta)$ when $N$ is 2-connected. 
Proposition 6.3. Let $f: N^{n} \rightarrow M^{2 n-1}$, where $N$ is 2-connected and $M$ is connected. There is an isomorphism of abelian groups

$$
\chi: \Omega_{1}(P(f, f) ; \zeta) \stackrel{\simeq}{\longrightarrow} \bigoplus_{\sigma \in \pi_{1}\left(M, m_{0}\right)} \mathbb{Z}_{2} \times \pi_{2}\left(M, m_{0}\right) .
$$

Proof. Since $N$ is 2-connected, the fibre inclusion $\iota: \Lambda M \rightarrow P(f, f)$ induces an isomorphism $\iota_{*}: \pi_{i}(\Lambda M) \rightarrow \pi_{i}(P(f, f))$ for $i=0,1$, and hence an isomorphism

$$
\iota_{*}: \Omega_{1}\left(\Lambda M ; \iota^{*} \zeta\right) \stackrel{\simeq}{\longrightarrow} \Omega_{1}(P(f, f) ; \zeta) .
$$

As noted in the proof of Lemma 5.1, the virtual bundle $\iota^{*} \zeta$ is trivial. Hence, we have isomorphisms

$$
\Omega_{1}\left(\Lambda M ; \iota^{*} \zeta\right) \cong \Omega_{1}^{f r}(\Lambda M) \cong \bigoplus_{\sigma \in \pi} \Omega_{1}^{f r}\left(\Lambda_{\sigma} M\right)
$$

where $\pi=\pi_{1}\left(M, m_{0}\right)$ and $\Omega_{*}^{f r}$ denotes the unreduced homology theory given by framed bordism (see [8] or [1]). The second isomorphism is given by the disjoint union axiom. The Atiyah-Hirzebruch spectral sequence for framed bordism gives a short exact sequence

$$
0 \rightarrow \mathbb{Z}_{2}=\Omega_{1}^{f r}(*) \rightarrow \Omega_{1}^{f r}\left(\Lambda_{\sigma} M\right) \rightarrow H_{1}\left(\Lambda_{\sigma} M ; \mathbb{Z}\right) \rightarrow 0
$$

which is split by the constant map $\Lambda_{\sigma} M \rightarrow *$. Since each path component $\Lambda_{\sigma} M$ is homotopy equivalent to the component $\Lambda_{0} M$ of the constant loop, we therefore have isomorphisms

$$
\Omega_{1}(P(f, f) ; \zeta) \cong \bigoplus_{\sigma \in \pi} \mathbb{Z}_{2} \times H_{1}\left(\Lambda_{0} M ; \mathbb{Z}\right) \cong \bigoplus_{\sigma \in \pi} \mathbb{Z}_{2} \times \pi_{2}\left(M, m_{0}\right)
$$

(by the Hurewicz homomorphism and the fact that $\pi_{1}\left(\Lambda_{0} M, m_{0}\right) \cong \pi_{2}\left(M, m_{0}\right)$ is abelian).

Hence, when $n \geq 5$ and $N$ is 2-connected, the image of a lift $\bar{\mu}(f)$ in a certain quotient of $\bigoplus_{\sigma \in \pi} \mathbb{Z}_{2} \times \pi_{2}\left(M, m_{0}\right)$ defines a complete obstruction to $f: N^{n} \rightarrow M^{2 n-1}$ being regularly homotopic to an embedding.

\section{REFERENCES}

1. J. P. Dax, Étude homotopique des espaces de plongements, Ann. Sci. École Norm. Super. 5(4) (1972), 303-377.

2. T. Ekholm, Regular homotopy and Vassiliev invariants of generic immersions $S^{k} \rightarrow$ $\mathbb{R}^{2 k-1}, k \geq 4$, J. Knot Theory Ramifications 7(8) (1998), 1041-1064.

3. A. Haefliger, Plongements différentiables dans le domaine stable, Comment. Math. Helv. 37 (1962), 155-176.

4. A. Hatcher and F. Quinn, Bordism invariants of intersections of submanifolds, Trans. Amer. Math. Soc. 200 (1974), 327-344.

5. A. Juhász, A geometric classification of immersions of 3-manifolds into 5-space, Manuscr. Math. 117(1) (2005), 65-83.

6. J. R. Klein and E. B. Williams, Homotopical intersection theory, I, Geom. Topol. 11 (2007), 939-977. 
7. J. R. Klein and E. B. Williams, Homotopical intersection theory, II: Equivariance, Math. Z. 264(4) (2010), 849-880.

8. U. Koschorke, Vector fields and other vector bundle morphisms-A singularity approach, Lecture Notes in Mathematics, vol. 847 (Springer, Berlin, 1981).

9. B. A. Munson, A manifold calculus approach to link maps and the linking number, Algebr. Geom. Topol. 8(4) (2008), 2323-2353.

10. O. Saeki, A. Szücs and M. Takase, Regular homotopy classes of immersions of 3manifolds into 5-space, Manuscr. Math. 108(1) (2002), 13-32.

11. K. Salikhov, Multiple points of immersions, preprint, arXiv:math/0203118

12. H. A. Salomonsen, Bordism and geometric dimension, Math. Scand. 32 (1973), 87-111.

13. A. Shapiro, Obstructions to the imbedding of a complex in a euclidean space. I. The first obstruction, Ann. Math. 66(2) (1957), 256-269. 256.

14. A. Szücs, Note on double points of immersions, Manuscr. Math. 76(3-4) (1992), 251-

15. C. T. C. Wall, Surgery of non-simply-connected manifolds, Ann. Math. 84(2) (1966), $217-276$.

16. C. T. C. Wall, Surgery on compact manifolds (Ranicki A. A., Editor), Mathematical Surveys and Monographs, vol. 69 (Amer. Math. Soc., Providence, RI, 1999).

17. H. Whitney, The self-intersections of a smooth $n$-manifold in $2 n$-space, Ann. Math. 45(2) (1944), 220-246. 